\title{
A sensitive fluorescent biosensor for the detection of copper ion inspired by biological recognition element pyoverdine
}

\author{
Kun Yin ${ }^{\mathrm{a}, \mathrm{b}}$, Yixuan $\mathrm{Wu}^{\mathrm{a}, \mathrm{b}}$, Shasha Wang ${ }^{\mathrm{a}, \mathrm{b}}$, Lingxin Chen ${ }^{\mathrm{a}, *}$ \\ a Key Laboratory of Coastal Environmental Processes and Ecological Remediation, Yantai Institute of Coastal Zone Research, Chinese Academy of Sciences, \\ Yantai 264003, China \\ ${ }^{\mathrm{b}}$ University of Chinese Academy of Sciences, Beijing 100049, China
}

\section{A R T I C L E I N F O}

\section{Article history:}

Received 18 December 2015

Received in revised form 18 March 2016

Accepted 24 March 2016

Available online 25 March 2016

\section{Keywords:}

Copper ion

Fluorescent biosensor

Pyoverdine

Electron transfer

\begin{abstract}
A B S T R A C T
The environmental copper pollution seriously threatens the health of organisms and the safety of ecosystem. Therefore, the development of a simple and sensitive method to detect copper ion is very important. In this study, we have developed a fluorescent biosensor based on biological recognition element pyoverdine to selectively detect copper ion. The fluorescence of pyoverdine is quenched obviously after binding with copper ion. A good linearity within the range of $0.2-10 \mu \mathrm{M}(\mathrm{R}=0.997)$ is attained and the detection limit is $50 \mathrm{nM}$. The biosensor has been successfully utilized for the detection of copper ion in drinking water, seawater and bio-samples and the results agree well with those obtained by the inductively coupled plasma mass spectrometry. Therefore, the established biosensor is a creditable method to detect copper ion with high sensitivity and selectivity, which can be utilized as a powerful tool to monitor copper pollution in the environment.
\end{abstract}

(c) 2016 Elsevier B.V. All rights reserved.

\section{Introduction}

Copper ion, as an important cofactor or structural component for various metal-coenzymes, plays critical roles in diverse biological physiological processes of organisms [1]. However, under overloading condition, copper ion can lead to a series of diseases such as Alzheimer's disease, Menkes disease and Wilson disease [2]. Additionally, the elevated copper ion level may also contribute to several cancers by activating oncogenic BRAF signal [3]. Considering the Janus-faced role of copper ion, its upper limit in drinking water is set at $20 \mu \mathrm{M}$ by U.S. Environmental Protection Agency [4]. However, the level of copper ion is usually much higher in polluted waters, which may seriously threaten the health of human beings [5]. Therefore, the credible detection of environmental copper ion is very necessary. To date, inductively coupled plasma-mass spectrometry (ICP-MS) and atomic absorption spectrometry (AAS) are the most widely used methods for copper ion determination $[6,7]$. In spite of the advantage of high-efficiency, these methods possess some inevitable shortcomings, such as long determination time and requirement of expensive and sophisticated equipment.

\footnotetext{
* Corresponding author.

E-mail address: lxchen@yic.ac.cn (L. Chen).
}

Therefore, the development of simple methods for the detection of trace copper ion has caused scientists' wide interest. Recently, various colorimetric sensors, electrochemical techniques and fluorescence probes have been established to detect copper ion [8-18]. The colorimetric sensors based on nanomaterials own outstanding advantages such as good sensitivity and can be directly recognized by naked eyes. However, some of the colorimetric sensors are easily influenced by complicated matrix. Compared with other techniques, fluorescence probes attract much attention for their high sensitivity and selectivity. And a myriad of functioned materials have been synthesized with extensive efforts to establish fluorescence probes. Among them, nanoparticles and fluorescent chromophores are the most widely used materials for their smallsize and specific molecular recognition [13-18]. However, the design and synthesis of these materials are a little complicated and difficult, which is usually a big challenge for developing new fluorescence probes. In comparison, biological recognition elements can be directly obtained from several organisms, which is much easier than synthetic probes. Additionally, biological recognition elements are smart for their special bio-functions as well as good biocompatibility. Hence, these biological recognition elements are suitable to develop novel, simple and selective fluorescence biosensors. 
Pyoverdine is a kind of extracellular siderophore of Pseudomonas aeruginosa, which can be largely secreted under iron-deficient conditions [19]. It helps Pseudomonas aeruginosa uptake iron to overcome iron limitation as well as protects them from heavy metal toxicity [20]. As the excellent fluorescence property of pyoverdine, it has already been utilized to established biosensors for the detection of dissociative ferric ion and furazolidone $[21,22]$. In this work, we have discovered that the fluorescence of pyoverdine can be quenched obviously in the presence of copper ion under neutral condition. Utilizing this phenomenon, a fluorescence biosensor based on pyoverdine for the detection of copper ion has been developed, which is much simpler than the previous reported methods. The detection of copper ion will not be influenced by dissociative ferric ion, because it only exists in acid environment. Additionally, furazolidone is almost nonexistent in the water and can be removed by ultraviolet digestion, which also will not influence the detection. Therefore, our biosensor can detect copper ion with excellent selectivity. Furthermore, our biosensor can detect copper ion with satisfied sensitivity, which has great potential to detect copper ion in real samples.

\section{Experimental section}

\subsection{Reagents and apparatus}

$\mathrm{KCl}, \mathrm{NaCl}, \mathrm{LiCl}, \mathrm{CaCl}_{2}, \mathrm{MgCl}_{2}, \mathrm{ZnCl}_{2}, \mathrm{Cd}\left(\mathrm{NO}_{3}\right)_{2}, \mathrm{MnCl}_{2}, \mathrm{~Pb}\left(\mathrm{NO}_{3}\right)_{2}$, $\mathrm{HgCl}_{2}$ and $\mathrm{FeCl}_{3}$ were purchased from Sinopharm Chemical Reagent Co., Ltd. (Shanghai, China). All other chemicals and metal salts used in this research were of analytic grade or better. Fluorescence change of pyoverdine in the absence/presence of copper ion was observed by naked eyes with triple UV analyzer WFH-203B (Jingke Industral Co., Ltd., Shanghai, China). And fluorescence intensity of pyoverdine in the absence/presence of copper ion was quantitatively measured by FluoroMax- 4 spectrofluorometer equipped with a xenon lamp (HORIBA Scientific, Japan).

\subsection{Pyoverdine purification}

Crude pyoverdine was obtained from Pseudomonas aeruginosa PA1, and the isolation method had been reported in our previous work [22]. The crude pyoverdine was firstly purified by copperchelate chromatography. Then the preliminary purified pyoverdine with $10 \mathrm{mM}$ EDTA was further purified by Sephadex G-15 column $(1.5 \times 100 \mathrm{~cm})$ [23]. The column was eluted by deionized water and fractions with the highest fluorescence intensity (excitation/emission at $410 / 460 \mathrm{~nm}$ ) were collected. The fractions were lyophilized to obtain pure pyoverdine. Then the purified pyoverdine was analyzed by HRMS spectroscopy. The molecular weight of purified pyoverdine was about 1278 (Fig. S7), which was similar with the previous researches [24,25]. The little difference was resulted from the variable peptide chain of pyoverdine, which could also be utilized as a fingerprint method to distinguish strains from each other [26]. Finally, the purified pyoverdine was dissolved in $10 \mathrm{mM}$ 4-(2-hydroxyerhyl) piperazine-1-erhanesulfonic acid (HEPES) buffer solution ( $\mathrm{pH} 7.0$ ) to $1 \mu \mathrm{g} / \mathrm{L}$ to detect copper ion.

\subsection{Detection of copper ion by pyoverdine}

Pyoverdine was incubated with copper ion in $50 \mathrm{mM}$ HEPES buffer solution $(\mathrm{pH} 7.0)$ at $25^{\circ} \mathrm{C}$ for $30 \mathrm{~min}$. The fluorescence intensity of the mixed solution was detected at the excitation wavelength of $410 \mathrm{~nm}$ with FluoroMax-4 spectrofluorometer.

\subsection{Selectivity and sensitivity for the copper ion detection}

To examine the selectivity of the biosensor, pyoverdine was incubated with $100 \mu \mathrm{M} \mathrm{Cu}{ }^{2+}, 1 \mathrm{mMK}^{+}, \mathrm{Na}^{+}, \mathrm{Li}^{+}, \mathrm{Ca}^{2+}, \mathrm{Mg}^{2+}, \mathrm{Zn}^{2+}$, $\mathrm{Cd}^{2+}, \mathrm{Mn}^{2+}, \mathrm{Pb}^{2+}, \mathrm{Hg}^{2+}$ and $\mathrm{Fe}^{3+}$ in $50 \mathrm{mM}$ HEPES buffer solution ( $\mathrm{pH}$ 7.0 ) at $25^{\circ} \mathrm{C}$ for $30 \mathrm{~min}$, respectively. To further explore it's specificity, the biosensor in the presence of $100 \mu \mathrm{M} \mathrm{Cu}^{2+}$ along with $100 \mu \mathrm{MK}^{+}, \mathrm{Na}^{+}, \mathrm{Li}^{+}, \mathrm{Ca}^{2+}, \mathrm{Mg}^{2+}, \mathrm{Zn}^{2+}, \mathrm{Cd}^{2+}, \mathrm{Mn}^{2+}, \mathrm{Pb}^{2+}, \mathrm{Hg}^{2+}$ and $\mathrm{Fe}^{3+}$ was incubated in $50 \mathrm{mM}$ HEPES buffer solution $(\mathrm{pH} \mathrm{7.0)}$ at $25^{\circ} \mathrm{C}$ for $30 \mathrm{~min}$, respectively. To investigate the linear range and detection limit of the biosensor, pyoverdine was incubated with different concentrations of copper ions $(0,0.5,1,2,4,6,8,10,20$, $40,60,80$ and $100 \mu \mathrm{M}$ ) in $50 \mathrm{mM}$ HEPES buffer solution ( $\mathrm{pH} 7.0$ ) at $25^{\circ} \mathrm{C}$ for $30 \mathrm{~min}$. The detection limit of the sensor was calculated by signal-to-noise $(\mathrm{S} / \mathrm{N})$ ratio equal to 3.0.

\subsection{Detection of copper ion in the spiked samples}

The drinking water sample was collected from municipal water supply system (Yantai, China). The seawater sample was collected from the Bohai Sea. The artificial cerebrospinal fluid was prepared followed the previous work [27]. All above samples were filtered by $0.22 \mu \mathrm{m}$ membrane for further use. The shellfish sample was purchased from Yanda market. The shellfish sample was washed by double deionized water $\left(\mathrm{ddH}_{2} \mathrm{O}\right)$ for three times and frozen at $-20^{\circ} \mathrm{C}$ in refrigerator for $12 \mathrm{~h}$. The shellfish sample was completely dried in a freeze-dryer and then grinded to powder. Next, $0.3 \mathrm{~g}$ powder sample was added into $10 \mathrm{~mL}$ concentrated nitric acid and incubated in a high pressure digestion tank at $150^{\circ} \mathrm{C}$ for $6 \mathrm{~h}$. After centrifuged, the supernatant was diluted to $50 \mathrm{~mL}$ by $\mathrm{ddH}_{2} \mathrm{O}$ and the $\mathrm{pH}$ was adjusted to $\mathrm{pH} 7.0$ by $1 \mathrm{M} \mathrm{NaOH}$. The $\mathrm{pH}$ value of all these samples was adjusted to $\mathrm{pH} 7.0$ by HEPES buffer before use. The concentrations of copper ion in these samples were detected both by our pyoverdine-based biosensor and ICP-MS, respectively.

\section{Results and discussion}

\subsection{Sensing principle of the biosensor for copper ion detection}

The proposed sensing mechanism of our biosensor for the detection of copper ion is shown in Scheme 1. Generally, the structure of pyoverdine is composed of three parts: a fluorescent dihydroxyquinoline chromophore, a variable peptide chain, and an acyl side chain. The metal-binding center is formed by the cooperation of dihydroxyquinoline and peptide chain, which can bind metal ions efficiently and further quench the fluorescence of pyoverdine. The quenching mechanism of the biosensor by copper ion is similar to some artificial fluorescence copper probes such as Compound 1 , and BODIPY $1[28,29]$. It is known that heavy metal ions can turn off the emission of fluorophore by their partially filled orbitals, unpaired electrons or heavy-atom effects, which quench the fluorescence through electron-transfer pathways [30]. In our reseach, copper ion can be captured by the hydroxyl and carbonyl group on pyoverdine and the electron transfer takes place between pyoverdine and copper ion, which further results in the fluorescence quenching of pyoverdine (Scheme 1). The fluorescence quenching of pyoverdine in the presence of copper ion can be quantitatively detected by FluoroMax-4 spectrofluorometer and the fluorescence quenching efficiency is calculated by $\left(F_{0}-F_{1}\right) / F_{0} \times 100 \%$, where $F_{0}$ and $F_{1}$ mean the fluorescence intensity of pyoverdine in the absence and presence of copper ion, respectively [31]. To further examine the quenching mechanism, we obtained the fluorescence quenching data referred to Stern-Volmer (SV) equation:

$\frac{F_{1}}{F_{0}}=1+K_{\mathrm{sv}}[Q]$ 


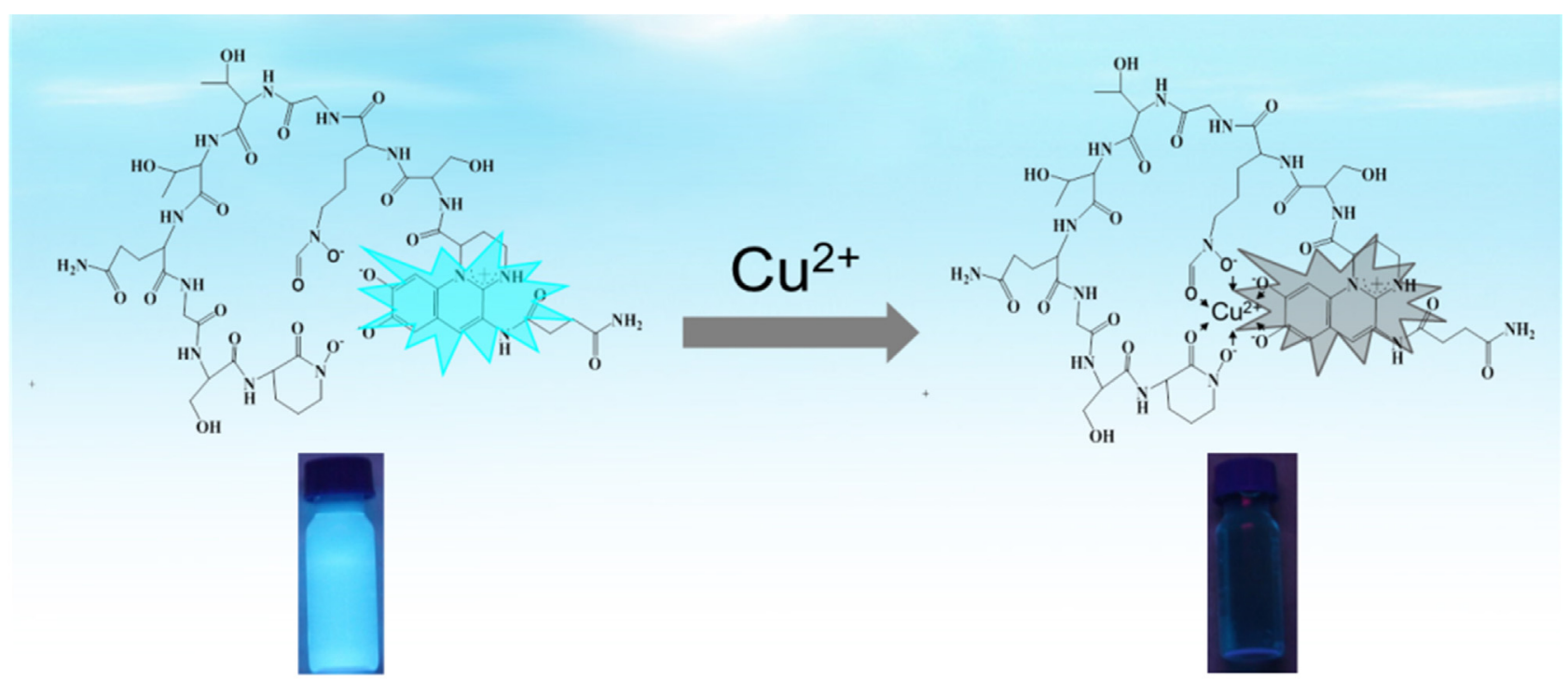

Scheme 1. Proposed mechanism for the detection of copper ion by pyoverdine.

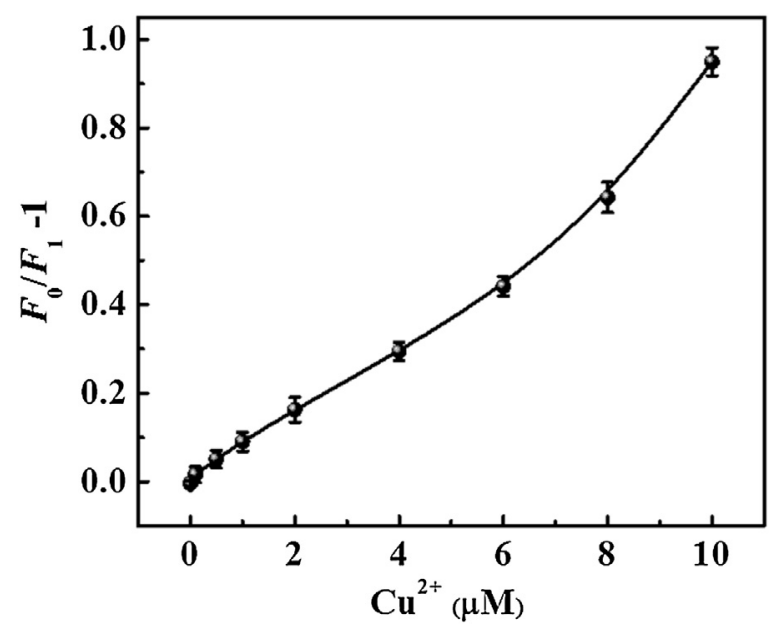

Fig. 1. The Stern-Volmer equation plots of pyoverdine in the presence of copper ion. The pyoverdine with different concentrations of copper ion $(0,0.1,0.5,1.0,2.0,4.0$, 8.0 and $10 \mu \mathrm{M}$ ) in $50 \mathrm{mM}$ HEPES solutions, pH 7.0 and incubated at $25^{\circ} \mathrm{C}$ for $30 \mathrm{~min}$. Data were the means for five independent experiments.

where $K_{\mathrm{sv}}$ meant the quenching constant and [Q] meant the concentration of copper ion. As shown in Fig. 1, the SV plots were nearly linear at low copper ion concentrations and tended to diverge from linearity and bended upwards with the concentration of copper ion increasing, which indicated a combination of dynamic and static quenching of pyoverdine in presence of copper ion [32]. Thus, the concentration of copper ion can be quantified by the fluorescent quenching efficiency of pyoverdine and a simple biosensor to detect copper ion is developed. Additionally, the fluorescence stability and photo stability of the pyoverdine-based biosensor were also investigated by us. As shown in Figs. S1 and S2, the biosensor owned satisfied fluorescence stability and photo stability, which was fitted for the detection of copper ion.

\subsection{The detection of copper ion by the biosensor}

To investigate the influence of copper ion on the fluorescence spectra of our biosensor, the purified pyoverdine with $100 \mu \mathrm{M}$ copper ion was incubated in $50 \mathrm{mM}$ HEPES solution at $25^{\circ} \mathrm{C}$ for $30 \mathrm{~min}$.

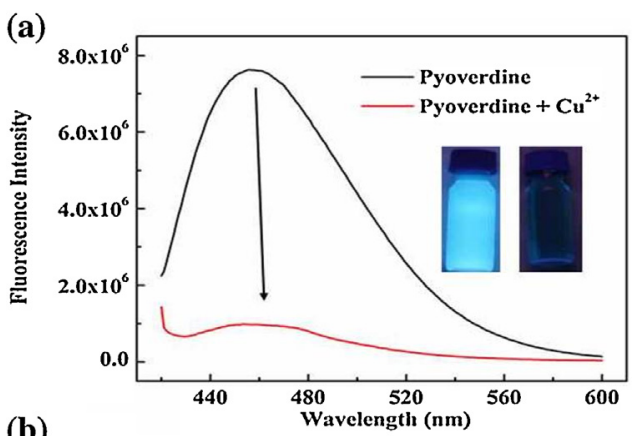

(b)

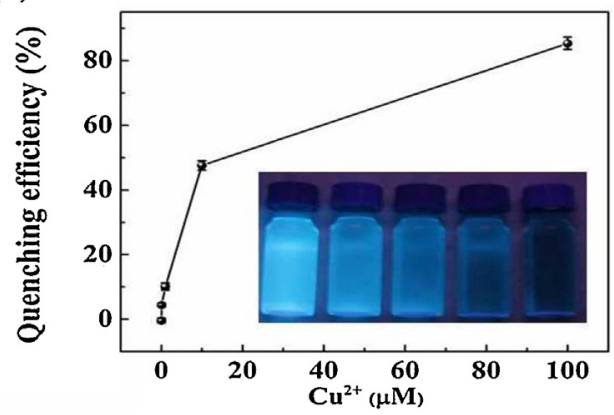

Fig. 2. (a) The fluorescence intensity of pyoverdine in the presence of $100 \mu \mathrm{M}$ copper ion. (b) The fluorescence quenching efficiency of pyoverdine in the presence of $0 \mathrm{nM}$, $100 \mathrm{nM}, 1 \mu \mathrm{M}, 10 \mu \mathrm{M}, 100 \mu \mathrm{M}$ copper ion. Bottles insert were watched directly by naked eyes under the triple UV analyzer at $325 \mathrm{~nm}$. Data were the means for five independent experiments.

As shown in Fig. 2a, the fluorescence intensity of pyoverdine was almost completely quenched in the presence of $100 \mu \mathrm{M}$ copper ion. According to Stern-Volmer equation, the fluorescent quenching efficiency of pyoverdine by $100 \mu \mathrm{M}$ copper ion could reach $82.5 \%$. Next, the biosensor was incubated with $100 \mathrm{nM}, 1 \mu \mathrm{M}, 10 \mu \mathrm{M}$ and $100 \mu \mathrm{M}$ copper ion in $50 \mathrm{mM}$ HEPES buffer solution (pH 7.0) at $25^{\circ} \mathrm{C}$ for $30 \mathrm{~min}$. The fluorescence of the biosensor was detected by the FluoroMax- 4 spectrofluorometer and identified by naked eyes with the help of triple UV analyzer at $325 \mathrm{~nm}$. As shown in Fig. 2b, the fluorescence quenching efficiency increased gradually with the concentration of copper ion increasing. And the fluorescence quenching of pyoverdine in the presence of $1 \mu \mathrm{M}$ copper ion 
could be distinguished clearly by naked eyes with the help of triple UV analyzer.

\subsection{Parameter optimization of the biosensor}

To investigate the optimal condition for the detection of copper ion by our biosensor, experimental parameters such as variety and concentration of buffer solution, $\mathrm{pH}$ and incubation time were taken into consideration. As buffer solution usually played an important role in the detection of copper ion, HEPES, B-R and PBS buffer solutions were investigated for their effects on the detection of copper ion by our biosensor. Pyoverdine with $10 \mu \mathrm{M}$ copper ion was added in different buffer solutions and incubated at $25^{\circ} \mathrm{C}$ for $30 \mathrm{~min}$. As shown in Fig. 3a, the response of our biosensor to copper ion was the best in $50 \mathrm{mM}$ HEPES buffer. And different concentrations of HEPES buffer showed no obvious influence on the performance of our biosensor (Fig. 3b). Next, we evaluated the effect of $\mathrm{pH}$ value on the quenching efficiency of copper ion to our biosensor. As shown in Fig. 3c, pH value from 5.0 to 9.0 showed little influence on the performance of our biosensor in the detection of copper ion. And $\mathrm{pH} 7.0$ was chosen for the following experiments because it was more convenient than detecting in acidic or alkaline environment and this $\mathrm{pH}$ value was similar to those of natural water samples. Additionally, the dissociative ferric ion could not interference the detection of copper ion at this $\mathrm{pH}$ value. Additionally, the detection of copper ion by the developed biosensor could be finished within $30 \mathrm{~min}$ (Fig. 3d). Therefore, the detection of copper ion by our biosensor was carried out in $50 \mathrm{mM}$ HEPES buffer, $\mathrm{pH} 7.0$ and incubated at $25^{\circ} \mathrm{C}$ for $30 \mathrm{~min}$.

\subsection{Sensitivity and selectivity of our biosensor for the detection of copper ion}

To verify the selectivity of the biosensor, pyoverdine with $100 \mu \mathrm{M} \mathrm{Cu}^{2+}, 1 \mathrm{mM} \mathrm{Na}^{+}, \mathrm{K}^{+}, \mathrm{Li}^{+}, \mathrm{Ag}^{+}, \mathrm{Ca}^{2+}, \mathrm{Zn}^{2+}, \mathrm{Mg}^{2+}, \mathrm{Mn}^{2+}, \mathrm{Cd}^{2+}$, $\mathrm{Hg}^{2+}, \mathrm{Pb}^{2+}$ and $\mathrm{Fe}^{3+}$ was incubated in $50 \mathrm{mM}$ HEPES buffer solution (pH 7.0) at $25^{\circ} \mathrm{C}$ for $30 \mathrm{~min}$, respectively. As shown in Fig. 4a, the fluorescence of pyoverdine was only quenched by copper ion, which could also be recognized by naked eyes. Additionally, we examined the quenching efficiency of $100 \mu \mathrm{M}$ copper ion to our biosensor in the presence of other ions, whose concentration was equal with that of copper ion. As shown in Fig. $4 \mathrm{~b}$, the quenching efficiency of copper ion would not be influenced by the coexisting ions. The previous research discovered that the fluorescence of pyoverdine could also be quenched by dissociative ferric ion under acidic condition $[21,33]$. Considering that the solubility product constant of ferric ion is $2.79 \times 10^{-39}$, the concentration of dissociative ferric ion is only $2.79 \times 10^{-18} \mathrm{M}$ under $\mathrm{pH}$ 7.0. We also examined the UV-vis absorption changes of pyoverdine-based biosensor toward copper ion and other metal ions. As shown in Fig. S3, the UV-vis absorption of pyoverdine was not be influenced by copper ion and other metal ions. Therefore, the detection of copper ion by our biosensor would not be influenced by dissociative ferric ion under our experiment condition. Additionally, we investigated the effects of sulphide, sulphate and other common anions on the detection of copper ion. As shown in Fig. S4a, the fluorescence of pyoverdine could not be influenced by sulphide, sulphate and other common anions. But the quenching efficiency of copper ion to pyoverdine could be influenced obviously by sulphide (Fig. S4b), because sulphide could react with copper ions to form a low-solubility product $\mathrm{CuS}\left(K_{\mathrm{sp}}=6.3 \times 10^{-36}\right)$ [34]. However, the sulphide and copper ion could not coexist in the environmental samples [35]. Therefore, the sulphide could not influence the detection of copper ion by our pyoverdine-based biosensor.

Next, we investigated the performance of our biosensor to quantitatively detect copper ion. Different concentrations of copper ion from 0.2 to $100 \mu \mathrm{M}$ were added and detected under optimal condition. As shown in Fig. 4a and b, a good linear correlation was obtained between the quenching efficiency and the concentration of copper ion from 0.2 to $10 \mu \mathrm{M}$. And the detection limit of biosensor pyoverdine to copper ion was up to $50 \mathrm{nM}$ (signal to noise ratio equal to 3.0), which was equal with the established copper ion fluorescence probes by polyamine-functionalized carbon quantum dots and pyoverdine-similar chemosensors [14,36,37]. The results indicated that the developed biosensor can detect copper ion with satisfied sensitivity. Additionally, we also investigated the reuse ability of the pyoverdine-based biosensor. As shown in S5, the fluorescence of pyoverdine could be recovered by $0.1 \mathrm{mM}$ EDTA. And then the pyoverdine could be regained by Sephadex G-15 column, which still could be utilized to detect copper ion. As shown in S6, the pyoverdine-based biosensor could be reused for at least 5 times.

\subsection{Applications of the biosensor to spiked samples}

To explore the applicability of our biosensor for practical sample analysis, copper ion in spiked water and biological samples was detected by our biosensor and traditional method ICP-MS, respectivey. As shown in Table. 1, the concentrations of copper ion in these samples were very low $(0.01 \mu \mathrm{M}$ in drinking water, $0.04 \mu \mathrm{M}$ in seawater, $0.03 \mu \mathrm{M}$ in shellfish samples and not detected in artificial cerebrospinal fluid samples), which could not be detected directly by our developed biosensor. However, the spiked copper in these samples could be detected by the developed biosensor and the results agreed well with those obtained by traditional method ICP-MS. Considering that the concentration of copper in the polluted area could be higher than $20 \mu \mathrm{M}$ [5]. Therefore, our developed pyoverdine-based biosensor could be utilized to detect copper ion in polluted water samples. Additionally, the concentration of cop-

Table 1

Comparison of the results obtained by our biosensor and ICP-MS for the detection of copper ion in spiked samples $(\mathrm{n}=5)$.

\begin{tabular}{|c|c|c|c|c|}
\hline Sample & $\begin{array}{l}\text { Added } \\
\text { Conc./ } \mu \mathrm{M}\end{array}$ & Detected $/ \mu \mathrm{M}$ & Recovery (100\%) & $\mathrm{ICP}-\mathrm{MS} / \mu \mathrm{M}$ \\
\hline \multirow[t]{3}{*}{ Drinking Water } & 0 & ND & ND & 0.01 \\
\hline & 5 & $4.89 \pm 0.21$ & $97.8 \pm 4.2$ & 5.13 \\
\hline & 10 & $10.12 \pm 0.46$ & $101.2 \pm 4.6$ & 9.96 \\
\hline \multirow[t]{3}{*}{ Seawater } & 0 & ND & ND & 0.04 \\
\hline & 5 & $5.17 \pm 0.19$ & $103.4 \pm 3.8$ & 4.98 \\
\hline & 10 & $9.69 \pm 0.32$ & $96.9 \pm 3.2$ & 10.14 \\
\hline \multirow[t]{3}{*}{ Shellfish } & 0 & ND & ND & 0.03 \\
\hline & 5 & $5.32 \pm 0.27$ & $106.4 \pm 5.4$ & 5.22 \\
\hline & 10 & $9.84 \pm 0.41$ & $98.4 \pm 4.1$ & 9.75 \\
\hline \multirow[t]{3}{*}{ Cerebrospinal Fluid } & 0 & ND & ND & ND \\
\hline & 5 & $5.16 \pm 0.41$ & $103.2 \pm 8.2$ & 5.27 \\
\hline & 10 & $10.17 \pm 0.89$ & $101.7 \pm 8.9$ & 10.39 \\
\hline
\end{tabular}

ND means not detectable. 
(a)

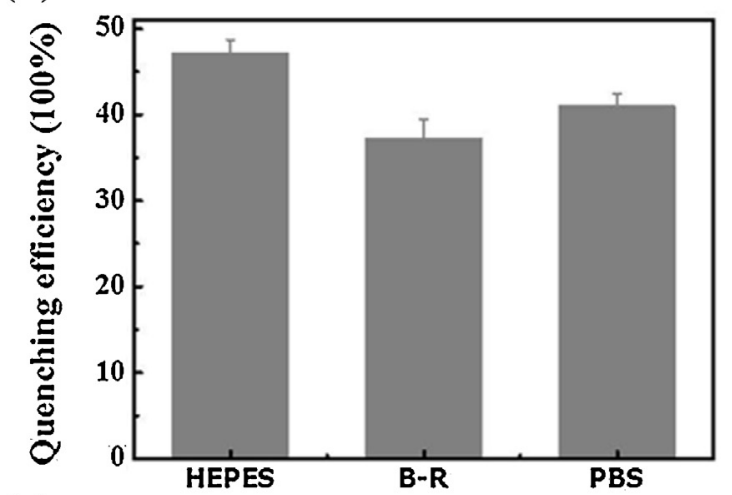

(c)

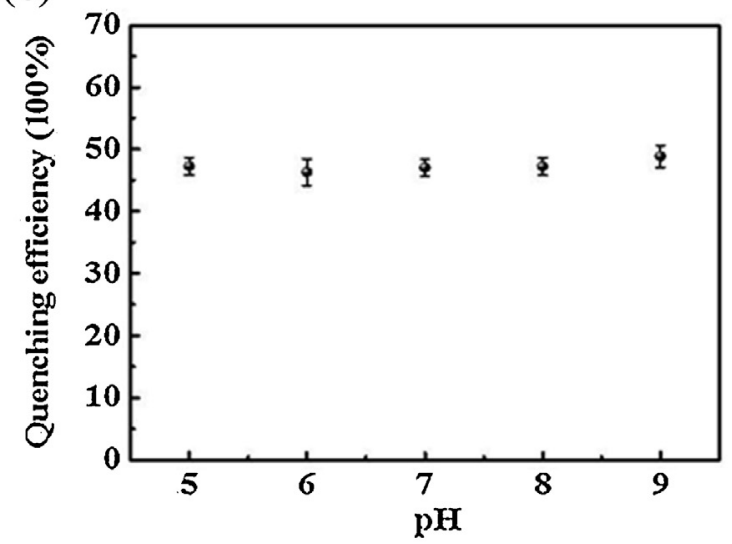

(b)
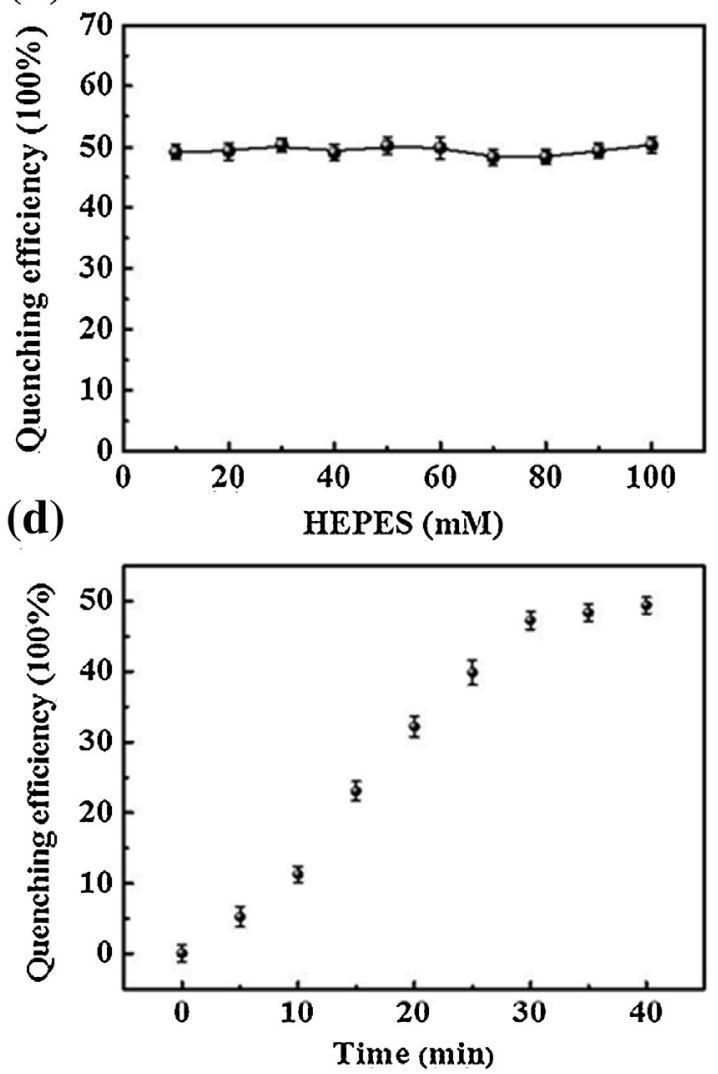

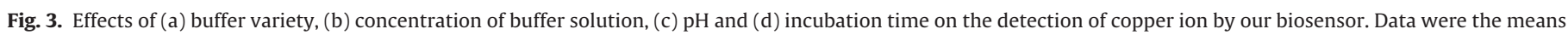
for five independent experiments.

(a)

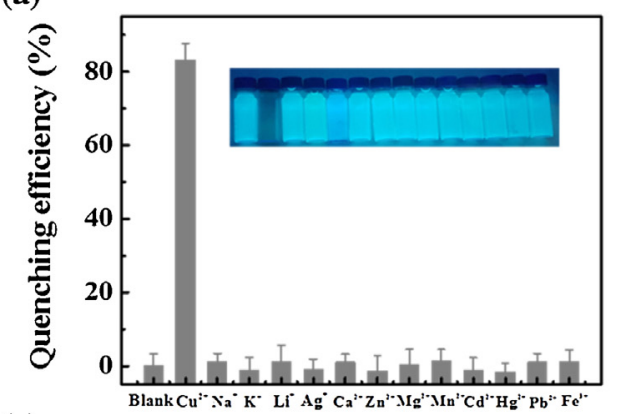

(c)

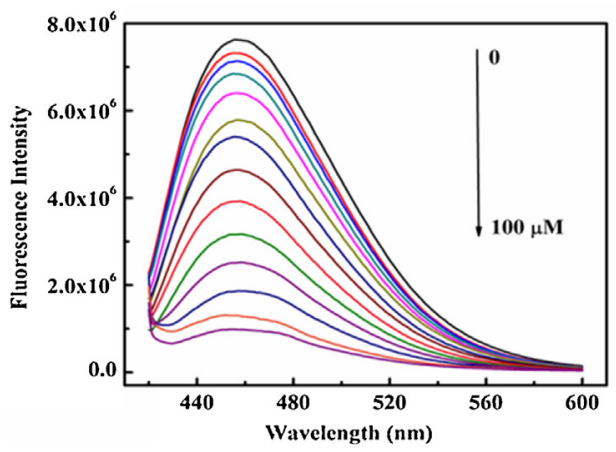

(b)

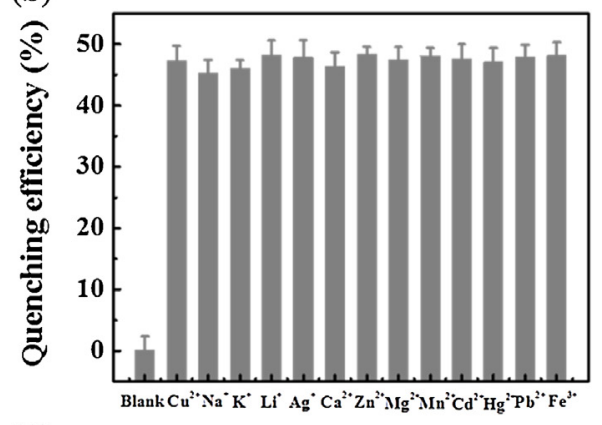

(d)

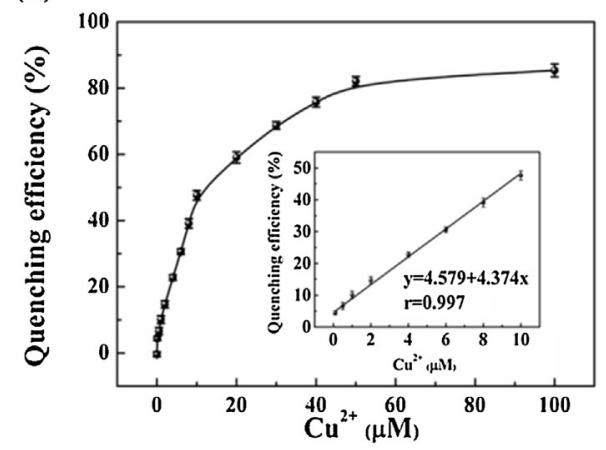

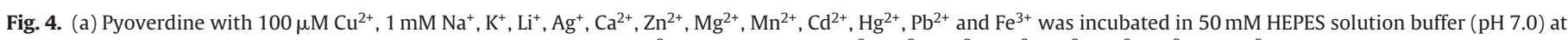

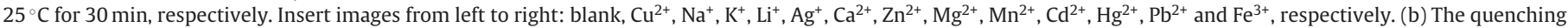

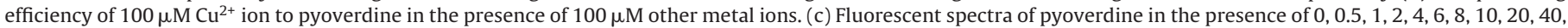

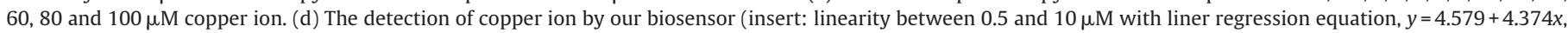
$r=0.997$, where ' $y$ ' represents the quenching efficiency and ' $x$ ' represents the concentration of copper ion). Data were the means for five independent experiments. 
per ion in cerebrospinal fluid would rise in patients of Parkinson's disease [38]. Therefore, our pyoverdine-based biosensor could also be used in disease prediction. All these results indicated that the developed biosensor were practically feasible for the detection of copper ion in real samples with good reliability and high accuracy.

\section{Conclusions}

We have developed an effective fluorescent biosensor for the detection of copper ion based on bio-element pyoverdine secreted by Pseudomonas aeruginosa PA1. The biosensor is designed based on copper-ion-induced fluorescence quenching of pyoverdine. Under optimal condition, the detection limit of our biosensor for copper ion is $50 \mathrm{nM}$. And $1 \mu \mathrm{M}$ copper ion can be distinguished directly by naked eyes under UV light. Additionally, the biosensor has been successfully applied to detect copper ion in spiked drinking water, seawater and bio-samples. Therefore, our biosensor owns outstanding advantages of high selectivity and sensitivity for copper ion detection and shows great potential application in the real sample analysis.

\section{Acknowledgments}

The research was financially supported by the National Natural Science Foundation of China (No. 21575159, 21275158), the Strategic Priority Research Program of the Chinese Academy of Sciences (XDA11020702).

\section{Appendix A. Supplementary data}

Supplementary data associated with this article can be found, in the online version, at http://dx.doi.org/10.1016/j.snb.2016.03.128.

\section{References}

[1] B. Reinhammar, R. Malkin, P. Jensen, B. Karlsson, L.E. Andréasson, R. Aasa, T. Vänngård, B. Malmström, A new copper(II) electron paramagnetic resonance signal in two laccases and in cytochrome c oxidase, J. Biol. Chem. 255 (1980) 5000-5003.

[2] L. Hou, M.G. Zagorski, NMR reveals anomalous copper(II) binding to the amyloid Abeta peptide of Alzheimer's disease, J. Am. Chem. Soc. 128 (2006) 9260-9261

[3] D.C. Brady, M.S. Crowe, M.L. Turski, G.A. Hobbs, X. Yao, A. Chaikuad, S. Knapp, K. Xiao, S.L. Campbell, D.J. Thiele, Copper is required for oncogenic BRAF signalling and tumorigenesis, Nature 509 (2014) 492-496.

[4] Edition of the Drinking Water Standards and Health Advisories [S], United States Environmental Protection Agency, 2012.

[5] J.O. Nriagu, Toxic metal pollution in Africa, Sci. Total Environ. 121 (1992) 1-37.

[6] J.W. Olesik, Elemental analysis using ICP-OES and ICP/MS, Anal. Chem. 63 (1991) 12-21.

[7] J. Chen, K.C. Teo, Determination of cadmium copper, lead and zinc in water samples by flame atomic absorption spectrometry after cloud point extraction, Anal. Chim. Acta 450 (2001) 215-222.

[8] T. Lou, L. Chen, Z. Chen, Y. Wang, L. Chen, J. Li, Colorimetric detection of trace copper ions based on catalytic leaching of silver-coated gold nanoparticles, ACS Appl. Mater. Interfaces 3 (2011) 4215-4220.

[9] S. Wang, Z. Chen, L. Chen, R. Liu, L. Chen, Label-free colorimetric sensing of copper (II) ions based on accelerating decomposition of $\mathrm{H}_{2} \mathrm{O}_{2}$ using gold nanorods as an indicator, Analyst 138 (2013) 2080-2084.

[10] K. Yin, B. Li, X. Wang, W. Zhang, L. Chen, Ultrasensitive colorimetric detection of $\mathrm{Cu}^{2+}$ ion based on catalytic oxidation of L-cysteine, Biosens. Bioelectron. 64 (2015) 81-87.

[11] L.P. Singh, J.M. Bhatnagar, Copper(II) selective electrochemical sensor based on Schiff Base complexes, Talanta 64 (2004) 313-319.

[12] E. Rossinyol, A. Prim, E. Pellicer, J. Rodríguez, F. Peiro, A. Cornet, J.R. Morantea, B. Tianb, T. Bob, D. Zhao, Mesostructured pure and copper-catalyzed tungsten oxide for $\mathrm{NO}_{2}$ detection, Sens. Actuators B-Chem. 126 (2007) 18-23.

[13] C. Yu, J. Zhang, J. Li, P. Liu, P. Wei, L. Chen, Fluorescent probe for copper (II) ion based on a rhodamine spirolactame derivative: and its application to fluorescent imaging in living cells, Microchim. Acta 174 (2011) 247-255

[14] C. Yu, J. Zhang, R. Wang, L. Chen, Highly sensitive and selective colorimetric and off-on fluorescent probe for $\mathrm{Cu}^{2+}$ based on rhodamine derivative, Org. Biomol. Chem. 8 (2010) 5277-5279.

[15] R. Krämer, Fluorescent chemosensors for $\mathrm{Cu}^{2+}$ ions: fast selective, and highly sensitive, Angew. Chem. Int. Ed. Engl. 37 (1998) 772-773.
[16] X. Xie, Y. Qin, A dual functional near infrared fluorescent probe based on the bodipy fluorophores for selective detection of copper and aluminum ions, Sen. Actuators B-Chem. 156 (2011) 213-217.

[17] Y.T. Su, G.Y. Lan, W.Y. Chen, H.T. Chang, Detection of copper ions through recovery of the fluorescence of DNA-templated copper/silver nanoclusters in the presence of mercaptopropionic acid, Anal. Chem. 82 (2010) 8566-8572.

[18] H.S. Jung, P.S. Kwon, J.W. Lee, J.I. Kim, C.S. Hong, J.W. Kim, S. Yan, J.Y. Lee, J.H. Lee, T. Joo, Coumarin-derived $\mathrm{Cu}(2+)$-selective fluorescence sensor: synthesis mechanisms, and applications in living cells, J. Am. Chem. Soc. 131 (2009) 2008-2012.

[19] S. Wendenbaum, P. Demange, A. Dell, J.M. Meyer, M. Abdallah, The structure of pyoverdine Pa, the siderophore of Pseudomonas aeruginosa Abdallah, Tetrahedron Lett. 24 (1983) 4877-4880.

[20] I.J. Schalk, M. Hannauer, A. Braud, New roles for bacterial siderophores in metal transport and tolerance, Environ. Microbiol. 13 (2011) 2844-2854.

[21] J. Barrero, C. Camara, M. Perez-Conde, C. San Jose, L. Fernandez, Pyoverdin-doped sol-gel glass for the spectrofluorimetric determination of iron (III), Analyst 120 (1995) 431-435.

[22] K. Yin, W. Zhang, L. Chen, Pyoverdine secreted by Pseudomonas aeruginosa as a biological recognition element for the fluorescent detection of furazolidone, Biosens. Bioelectron. 51 (2014) 90-96.

[23] R. Xiao, W.S. Kisaalita, Fluorescent pseudomonad pyoverdines bind and oxidize ferrous ion, Appl. Environ. Microbiol. 64 (1998) 1472-1476.

[24] J.G. Owen, D.F. Ackerley, Characterization of pyoverdine and achromobactin in Pseudomonas syringae pv. phaseolicola 1448, BMC Microbial. 11 (2011) $1471-2180$

[25] M. Hannauer, M. Schäfer, F. Hoegy, P. Gizzi, P. Wehrung, G.L. Mislin, H. Budzikiewicz, I.J. Schalk, Biosynthesis of the pyoverdine siderophore of Pseudomonas aeruginosa involves precursors with a myristic or a myristoleic acid chain, FEBS Lett. 586 (2012) 96-101.

[26] H. Eghbali, S. Matthijs, V. Verdoold, H. Gardeniers, P. Cornelis, G. Desmet, Use of non-porous pillar array columns for the separation of Pseudomonas pyoverdine siderophores as an example of a real-world biological sample, J. Chromatogr. A 1216 (2009) 8603-8611.

[27] B.M. Stell, S.G. Brickley, C.Y. Tang, M. Farrant, I. Mody, Neuroactive steroids reduce neuronal excitability by selectively enhancing tonic inhibition mediated by $\delta$ subunit-containing GABAA receptors, Proc. Natl. Acad. Sci. U. S. A. 100 (2003) 14439-14444.

[28] X. Qi, E.J. Jun, L. Xu, S.J. Kim, J.S. Hong, Y.J. Yoon, J. Yoon, New BODIPY derivatives as OFF-ON fluorescent chemosensor and fluorescent chemodosimeter for $\mathrm{Cu}^{2+}$ : cooperative selectivity enhancement toward $\mathrm{Cu}^{2+}$, J. Org. Chem. 71 (2006) 2881-2884.

[29] L. Zeng, E.W. Miller, A. Pralle, E.Y. Isacoff, C.J. Chang, A selective turn-on fluorescent sensor for imaging copper in living cells, J. Am. Chem. Soc. 128 (2006) 10-11.

[30] J. Chan, S.C. Dodani, C.J. Chang, Reaction-based small-molecule fluorescent probes for chemoselective bioimaging, Nat. Chem. 4 (2012) 973-984.

[31] R.L. Joseph, R. Lakowicz, Principles of Fluorescence Spectroscopy, Kluwer Academic/Plenum Publishers, New York, 1999, pp. 11.

[32] K. Yin, F. Yu, D. Liu, Z. Xie, L. Chen, Cyanine-based colorimetric and fluorescent probe for the selective detection of diethylstilbestrol in seawater, shrimp and fish samples, Sens. Actuators B-Chem. 223 (2016) 799-805.

[33] M.F. Yoder, W.S. Kisaalita, Iron specificity of a biosensor based on fluorescent pyoverdin immobilized in sol-gel glass, J. Biol. Eng. 5 (2011) 4.

[34] Y.F. Zhu, D.H. Fan, W.Z. Shen, A general chemical conversion route to synthesize various ZnO-based core/shell structures, J. Phys. Chem. C. 112 (2008) 10402-10406.

[35] H. Hirata, K. Date, Lead sulfide-impregnated silicone rubber membranes as selective electrodes for lead ion, Anal. Chem. 43 (1971) 279-281.

[36] Y. Dong, R. Wang, G. Li, C. Chen, Y. Chi, G. Chen, Polyamine-functionalized carbon quantum dots as fluorescent probes for selective and sensitive detection of copper ions, Anal. Chem. 84 (2012) 6220-6224.

[37] Y. Xiang, A. Tong, P. Jin, Y. Ju, New fluorescent rhodamine hydrazone chemosensor for $\mathrm{Cu}(\mathrm{II})$ with high selectivity and sensitivity, Org. Lett. 8 (2006) 2863-2866.

[38] H.S. Pall, D.R. Blake, J.M. Gutteridge, A.C. Williams, J. Lunec, M. Hall, A. Taylor, Raised cerebrospinal-fluid copper concentration in Parkinson's disease, Lancet 330 (1987) 238-241.

\section{Biographies}

Kun Yin received his BS degree in aquaculture from China Agriculture University He is currently a Ph.D. degree student at Yantai Institute of Coastal Zone Research, Chinese Academy of Sciences. His scientific interest includes bioremediation and biosensor for environmental pollutants.

Yixuan Wu received her BS degree in chemistry from University of Jinan, China in 2014. During the same year, he joined as a research assistant in the Laboratory of Environmental Microanalysis and Monitoring in Yantai Institute of Coastal Zone Research, Chinese Academy of Sciences. His current research interests focus on bioremediation for environmental pollutants.

Shasha Wang received her BS degree in chemistry from Qufu Normal University, China in 2010. During the same year, she joined the Laboratory of Environmental Microanalysis and Monitoring in Yantai Institute of Coastal Zone Research, Chinese 
Academy of Sciences. She is studying for her $\mathrm{PhD}$ degree. Her current research interests focus on investigation of optical sensors for environmental analysis using novel nanomaterials.

Lingxin Chen received his PhD degree in analytical chemistry from the Dalian Institute of Chemical Physics, Chinese Academy of Sciences, Dalian, in 2003. After 2 years of postdoctoral experience at the Department of Chemistry, Tsinghua University,
Beijing, he joined first as a BK21 researcher and then as a research professor at the Department of Applied Chemistry, Hanyang University, Korea, in 2006. In 2009, as a professor, he joined the Yantai Institute of Coastal Zone Research, Chinese Academy of Sciences, Yantai. His research interests include the studies of novel properties of materials such as functionalized nanoparticles for developing nanoscale biochemical analysis methods and molecular imprinting-based sample pretreatment technology. 